\title{
Mx2 expression is associated with reduced susceptibility to HIV infection in highly exposed HIV seronegative Kenyan sex workers
}

\author{
Derek R. Stein ${ }^{a, e}$, Souradet Y. Shaw ${ }^{b}$, Lyle R. McKinnon', Max Abou ${ }^{e}$, \\ Stuart J. McCorrister ${ }^{f}$, Garrett R. Westmacott ${ }^{f}$, Keith R. Fowke ${ }^{a}$, \\ Francis A. Plummer ${ }^{\mathrm{a}}$ and T. Blake Ball ${ }^{\mathrm{a}, \mathrm{c}, \mathrm{e}}$
}

\begin{abstract}
Introduction: Recent studies have identified Mx2 as a novel HIV-1 innate restriction factor that inhibits proviral integration. A pilot proteomic study of immune cells from highly exposed HIV-seronegative (HESN) individuals enrolled in the Pumwani sex worker cohort identified Mx1 as potential correlate of HIV protection. A detailed population level analysis of $M \times 1$ and $M \times 2$ expression and their role in reduced susceptibility to HIV infection in HESN women was conducted.

Methods: Peripheral blood mononuclear cells (PBMC) were isolated from $102 \mathrm{HESN}$ women and 100 high-risk negative controls enrolled in a Nairobi-based sex worker cohort. Whole-cell lysates were prepared and analyzed for Mx1 and Mx2 expression by commercial ELISA. Bivariate and multiple linear regression analyses were conducted to account for confounding epidemiological factors.
\end{abstract}

Results: Mx2, but not Mx1, was found to be significantly overexpressed in HESN women compared with high-risk negative controls $(P=0.027)$. After multiple linear regression analysis, accounting for age, menopause, pregnancy, Depo-Provera (Pfizer, New York, USA) use, recent infections and medication usage, Mx2 expression remained significantly overexpressed in the PBMC of HESN women $(P=0.05)$. Additionally, an interaction model analysis indicated that HESN women who use Depo-Provera (Pfizer, New York, USA) have 2.6-fold higher levels of Mx2 than any other group $(P<0.001)$. No associations with $M \times 1$ expression were observed.

Conclusion: This is the first epidemiological report of $M \times 2$ and its association with altered susceptibility to HIV infection in HESN women. Additionally, we show that HESN women who use Depo-Provera (Pfizer, New York, USA) have the highest levels of Mx2 expression, highlighting a possible mechanism for hormonal modulation of HIV susceptibility.

๑ 2014 Wolters Kluwer Health | Lippincott Williams \& Wilkins

AIDS 2015, 29:35-41

Keywords: Depo-Provera, DMPA, HIV, highly exposed HIV-seronegative, highly exposed HIV seronegative, Mx2

\footnotetext{
${ }^{a}$ Departments of Medical Microbiology, ${ }^{b}$ Community Health Sciences, ${ }^{\mathrm{C}}$ Immunology University of Manitoba, Winnipeg, Manitoba, Canada, ${ }^{\mathrm{d} C e n t e r}$ for the AIDS Programme of Research in South Africa (CAPRISA), Nelson R. Mandela School of

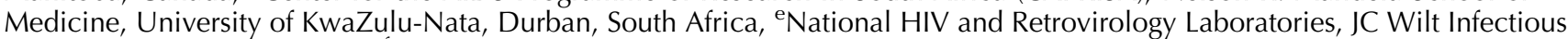
Disease Research Centre, and 'Mass Spectrometry and Proteomics Core Facility, Public Health Agency of Canada, Winnipeg, Manitoba, Canada.

Correspondence to Derek R. Stein, University of Manitoba, 543-745 Bannatyne Avenue Winnipeg, MB, Canada, R3E 0J9.

Tel: +1 204470 1910; e-mail: Derek.Riley.Stein@outlook.com

Received: 29 July 2014; revised: 10 September 2014; accepted: 16 September 2014.
}

DOI:10.1097/QAD.0000000000000490

ISSN 0269-9370 @ 2014 Wolters Kluwer Health | Lippincott Williams \& Wilkins Copyright (C) Lippincott Williams \& Wilkins. Unauthorized reproduction of this article is prohibited. 


\section{Introduction}

We have previously described a cohort of highly exposed HIV-seronegative (HESN) sex workers from Nairobi, Kenya, who can be epidemiologically defined as relatively resistant to HIV infection [1]. The phenotype of these HESN women has been shown to associate with altered interferon signaling mediated impart by interferon regulatory transcription factor-1 [2], T-cell immune quiescence [3], and using unbiased proteomic techniques, the altered expression of antiproteases in genital secretions $[4,5]$. However, a proteomics analysis of mucosal and systemic immune cells in the context of this rare phenotype has yet to be undertaken. In a pilot proteomics study of peripheral blood mononuclear cells (PBMC) isolated from HESN $(n=12)$ and HIV-susceptible controls $(n=18)$, we identified decreased expression of Mx1 $(P=0.029)$ as a potential marker of HIV susceptibility in HESN women (Fig. 1a). Validation of Mx1 by western blot and correlation analysis $(r=0.6772$, $P=0.0001)$ confirmed the lower $\mathrm{Mx} 1$ expression in the same HESN samples (Fig. 1b and c). The reduced expression of $\mathrm{Mx} 1$, and possibly other members of this family in HESN women, may reflect the altered interferon responsiveness previously observed in these individuals [6]. Additionally, recent studies have shown that $\mathrm{Mx} 2$ is a correlate of simian immunodeficiency virus (SIV) protection in macaques [7], restricts HIV-1

(a)

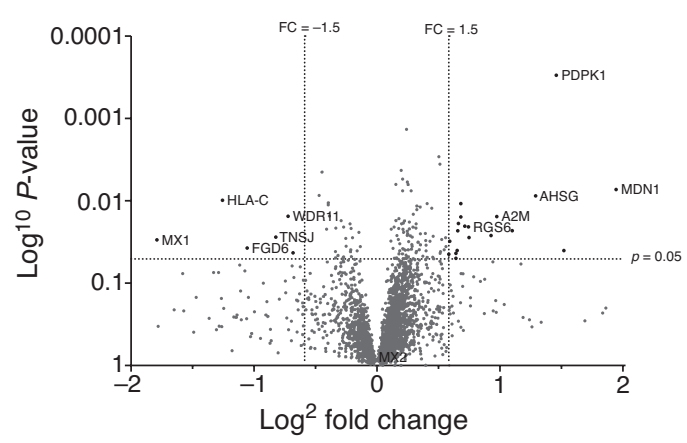

(b)

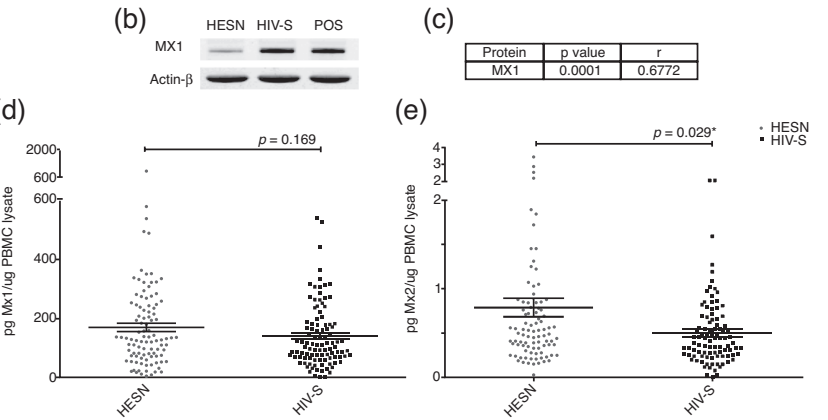

(d) infection in vitro [8-10], and is likely due to the destabilization of viral replication complexes or nuclear import [11]. Although $\mathrm{Mx} 2$ was not differentially regulated in our initial pilot data (Fig. 1a), these recent studies and the similar amino acid homology between the two proteins $(\sim 63 \%)$, led us to conduct a more detailed population level analysis of Mx1 and $\mathrm{Mx} 2$ expression in HESN women from the Pumwani sex worker cohort.

\section{Results}

To determine the expression levels of $\mathrm{Mx} 1$ and $\mathrm{Mx} 2$ in a larger study accounting for epidemeological covariates, we isolated PBMC from 102 HESN women meeting our epidemiologic definition of HIV resistance and 100 highrisk HIV-susceptible female sex workers (HIV-S). HESN women are epidemiologically defined as being active in sex work while remaining HIV negative (serology and PCR) for 7 years or longer. Given the volume of clients, the estimated prevalence of HIV in this population, and duration of seronegative follow-up, it is clear these individuals have intense exposure to HIV. A commercial ELISA was used to measure the levels of $\mathrm{Mx} 1$ and $\mathrm{Mx} 2$ in PBMC lysate from the two study groups. Bivariate comparisons indicated that the Mx1 expression in PBMC was similar between HESN women and HIV-S controls (Fig. 1d, $P=0.169)$, clearly in disagreement with our

(f)

\begin{tabular}{|c|c|c|c|c|c|c|}
\hline & No. & Mean & SD & Median & IQR & $\mathrm{p}$ \\
\hline Total & 161 & -0.80 & -0.80 & -0.80 & $-1.30--0.35$ & \\
\hline HESN vs. HIV-S (ref) & & & & & & 0.029 \\
\hline HIV-S & 78 & -0.95 & -0.75 & -0.92 & $-1.34--0.38$ & \\
\hline HESN & 83 & -0.67 & -0.82 & -0.72 & $-1.12--0.24$ & \\
\hline Age & & & & & & 0.098 \\
\hline$<34$ & 81 & -0.72 & -0.79 & -0.77 & $-1.26--0.17$ & \\
\hline $35-39$ & 35 & -0.73 & -0.81 & -0.67 & $-1.40--0.37$ & \\
\hline $40+$ & 45 & -1.02 & -0.78 & -0.90 & $-1.27--0.62$ & \\
\hline Contraception & & & & & & 0.018 \\
\hline None & 48 & -0.94 & -0.74 & -0.92 & $-1.35--0.38$ & \\
\hline Oral & 17 & -0.74 & -0.63 & -0.71 & $-1.41--0.19$ & \\
\hline DMPA & 31 & -0.44 & -0.96 & -0.56 & $-0.89--0.00$ & \\
\hline Condom & 48 & -0.88 & -0.67 & -1.04 & $-1.34--0.42$ & \\
\hline Depo-provera (DMPA) & & & & & & 0.005 \\
\hline Yes & 130 & -0.89 & 0.73 & -0.89 & $-1.36--0.37$ & \\
\hline No & 31 & -0.44 & 0.96 & -0.57 & $-0.88--0.00$ & \\
\hline Gravida & & & & & & 0.045 \\
\hline 0 & 88 & -0.66 & 0.81 & -0.70 & $-1.04--0.17$ & \\
\hline $1-2$ & 30 & -1.01 & 0.73 & -1.03 & $-1.42--0.37$ & \\
\hline $3+$ & 43 & -0.95 & 0.78 & -1.01 & $-1.34--0.48$ & \\
\hline Sex work duration & & & & & & 0.194 \\
\hline $2<$ years & 39 & -0.99 & 0.77 & -1.06 & $-1.45--0.57$ & \\
\hline $2-5$ years & 39 & -0.81 & 0.84 & -0.79 & $-1.36--0.23$ & \\
\hline $6+$ years & 83 & -0.71 & 0.78 & -0.78 & $-1.12--0.28$ & \\
\hline Sex work break & & & & & & 0.299 \\
\hline No & 104 & -0.76 & 0.81 & -0.80 & $-1.30--0.21$ & \\
\hline Yes & 57 & -0.89 & 0.78 & -0.88 & $-1.24--0.54$ & \\
\hline Infection, last 2 months & & & & & & 0.387 \\
\hline No & 135 & -0.78 & 0.80 & -0.79 & $-1.32--0.26$ & \\
\hline Yes & 26 & -0.93 & 0.78 & -0.98 & $-1.23--0.42$ & \\
\hline Medication, last 6 months & & & & & & 0.473 \\
\hline No & 135 & -0.78 & 0.84 & -0.79 & $-1.32--0.26$ & \\
\hline Yes & 26 & -0.91 & 0.50 & -0.90 & $-1.26--0.60$ & \\
\hline
\end{tabular}

Fig. 1. Expression of Mx1 and Mx2 in highly exposed HIV-seronegative women. (a) Protein biomarkers of interest differentially regulated between highly exposed HIV-seronegative (HESN) and HIV-susceptible controls $(P<0.05$ and a FC $>1.5)$. (b) Representative western blot analysis at the patient level with an HIV-infected individual included for comparative purposes and (c) correlation between the mass spectrometry and western blot expression. Expression levels are illustrated for (d) Mx1 and (e) Mx2 measured by commercial ELISA in $102 \mathrm{HESN}$ and 100 HIV-susceptible controls. (f) Bivariate analysis of epidemiological factors with respect to log Mx2 expression, excluding pregnant and postmenopausal women. 
proteomics pilot study. These discrepancies highlight the issues associated with accurately controlling for epidemiological confounders in proteomics or other systems biology approaches that are often found in small-scale human studies. Surprisingly, in contrast, $\mathrm{Mx} 2$ expression was found to be elevated in HESN women $(P=0.029)$ compared to HIV-S controls (Fig. 1e). Univariate analysis of epidemiologic confounders in these women showed factors such as age, duration of sex work, a break from sex work, and recent infections or medication use did not affect the expression of Mx2 (Fig. 1f) or Mx1 (Suppl. Table 1, http://links.lww.com/QAD/A590). However, when we examined contraception use, we found that women who reported using injectable Depo-Provera (DMPA; Pfizer, New York, USA) had significantly higher expression of $\mathrm{Mx} 2$ (Fig. 1f; $P=0.005$ ) compared with those who did not use DMPA.

To better define the relationship between DMPA use and systemic cellular expression of $\mathrm{Mx} 1$ and $\mathrm{Mx} 2$, multiple linear regression analysis was conducted to account for epidemiological confounders. In a multiple linear regression model adjusted for DMPA use, age, and current infections, the increased $\mathrm{Mx} 2$ expression associated with HESN women remained significant (Fig. 2a; $P=0.05$; model 3). An interaction indicator model between DMPA use and HESN status was also tested (Fig. 2a, model 4), and found that HESN women who use DMPA accounted for the majority of increased $\mathrm{Mx} 2$ expression observed in this group. The mean $\mathrm{Mx} 2$ expression in the PBMC of HESN women reporting DMPA use was on average 2.6-fold higher $(1.35 \rho \mathrm{g} / \mu \mathrm{g}$ of PBMC lysate) than any other group (Fig. 2 c; $P<0.0001$ ). Using the same linear regression analysis and interaction model no significant relationships for $\mathrm{Mx} 1$ were noted (Fig. 2b \& Suppl. Table 2, http://links.lww.com/QAD/ A590). Together these findings suggest an association between the HESN phenotype, DMPA use, and Mx2, but not Mx1 expression.

(a)

\begin{tabular}{lcccrr}
\hline & Crude & Model 1 & Model 2 & Model 3 & \multicolumn{2}{c}{ Model 4 } \\
\hline HESN vs. HIV-S (ref) & 0.277 & 0.246 & 0.249 & 0.241 & 0.119 \\
& $(0.03)^{\star}$ & $(0.05)^{\star}$ & $(0.04)^{\star}$ & $(0.05)^{\star}$ & $(0.39)$ \\
Depo-provera (DMPA) & & 0.418 & 0.369 & 0.369 & -0.028 \\
& & $(0.01)^{\star *}$ & $(0.02)^{\star}$ & $(0.02)^{\star}$ & $(0.91)$ \\
Age: 35-39 years (<35 years as ref) & & & 0.036 & 0.040 & 0.078 \\
& & & $(0.82)$ & $(0.80)$ & $(0.62)$ \\
Age: 40+ years (<35 years as ref) & & & -0.225 & -0.223 & -0.191 \\
& & & $(0.13)$ & $(0.13)$ & $(0.20)$ \\
Recent infection, last 2 months & & & & -0.035 & -0.009 \\
HESN and DMPA interation & & & & $(0.84)$ & $(0.96)$ \\
& & & & & 0.690 \\
$R^{2}$ & 0.03 & 0.07 & 0.09 & 0.09 & 0.12 \\
$N$ & 161 & 161 & 161 & 161 & 161 \\
\hline
\end{tabular}

(b)

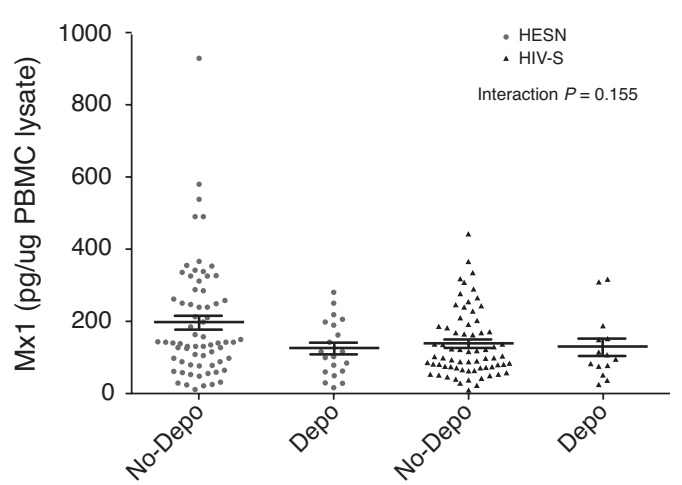

\section{Methods}

\section{Cohort participants}

The Pumwani sex worker cohort is an open cohort that provides HIV-1 prevention services and treatment through funding from the Presidents Emergency Plan 
For AIDS Relief. The cohort has enrolled over more than 3800 female sex workers, with approximately 250 new enrolments per year. These women are at extremely high risk of HIV-1 infection, with $50 \%$ of women being HIV-1 seropositive at enrollment. This cohort includes 1285 women in active follow-up, with 671 infected and 614 uninfected, of which 107 HESN participants meet our epidemiologic definition of resistance to HIV (HIV-R) infection ( $>7$ years in the cohort, active in sex work, and HIV-1 negative by ELISA and DNA/RNA testing). Women who are newly enrolled in the cohort, with 3 years or less of follow-up (HIV-S) are considered to be at the same risk of acquiring HIV infection as HIV-R women and act as an HIV-S control. Based upon 27 years of epidemiologic follow-up, we expect $85 \%$ of the HIV-S to seroconvert, despite strong HIV-1 prevention programmes. Both HIV-R and HIV-S have similar numbers of clients per day, as well as reported condom use with casual and regular clients. Written informed consent was obtained from all study participants of the Pumwani cohort. Approval for all studies was obtained from the research ethics board of the University of Manitoba, Winnipeg, Canada and the Kenyatta National Hospital, Nairobi, Kenya.

\section{Sample preparation}

PBMC were isolated for the pilot mass spectrometry and epidemiological studies by density gradient centrifugation (Ficoll-Paque). PBMC samples for the epidemiological studies conducted on $\mathrm{Mx} 1$ and $\mathrm{Mx} 2$ in the Pumwani cohort were isolated in Nairobi, Kenya, from 1995 to 2009. Sample storage length was found not to have any effect on $\mathrm{Mx} 1 / 2$ protein expression as well as cell viability by correlation analysis. PBMC were frozen in freezing media and shipped back to Winnipeg, Manitoba, Canada, and stored in liquid nitrogen. PBMCs were removed from the liquid nitrogen tank and rapidly thawed in a $37^{\circ} \mathrm{C}$ water bath. Cells were transferred from the cryovial and immediately placed in $10 \mathrm{ml}$ of RPMI supplemented with $10 \%$ fetal calf serum, penicillin, streptomycin, and fungizone (Life Technologies, Carlsbad, California, USA). Cell viability and counts were conducted on each sample to ensure a viability of at least 80\% using the Invitrogen Countess Automated Cell Counter (Carlsbad, California, USA) by trypan blue staining. After counting, the cells were centrifuged at $800 \mathrm{~g}$ for $10 \mathrm{~min}$. Cells were washed in PBS three times. Cells were then resuspended in either $200 \mu l$ of $4 \%$ SDS (MS analysis) or $500 \mu \mathrm{l}$ of PBS (ELISA analysis). PBMCs suspended in PBS were subjected to three rapid freeze thaw cycles on dry ice. The resulting PBMC lysate was centrifuged at $1500 \mathrm{~g}$ to remove any excess cellular debris. PBMCs suspended in 4\% SDS were immediately boiled for $5 \mathrm{~min}$ followed by centrifugation at $1500 \mathrm{~g}$ to remove any excess cellular debris. PBMC lysate was transferred to a new cryovial and stored at $-80^{\circ} \mathrm{C}$ for future use by mass spectrometry or ELISA analysis. The total protein concentration of PBMC lysate in $4 \%$ SDS and PBS was determined by 2D-Quant (GE Healthcare, Chalfont, UK) and BCA assay (Millipore, Billerica, Massachusetts, USA), respectively.

\section{Isobaric tags for relative and absolute quantitation mass spectrometry}

Trypsin digestion of PBMC lysate in 4\% SDS was performed by the filter-aided sample preparation method [12] with modifications, briefly described as follows: approximately $500 \mu \mathrm{g}$ of protein per digestion was mixed with $7 \times$ its volume in urea exchange buffer, then passed through a $10 \mathrm{kDa}$ Nanosep spin filter (Pall Corp., Port Washington, New York, USA). Two additional washes with UEB were performed to remove excess SDS. Protein was then alkylated with $50 \mathrm{mmol} / \mathrm{l}$ iodoacetamide for $20 \mathrm{~min}$ at room temperature in the dark. Filters were washed twice with UEB, treated with 1000 units of benzonase (Novagen, Madison, Wisconsin, USA) in $50 \mathrm{mmol} / 1$ Tris or $50 \mathrm{mmol} / 1 \mathrm{HEPES}$ for $30 \mathrm{~min}$ at room temperature, then rinsed again with Tris or HEPES buffer. Each filter was treated with $1.5 \mu \mathrm{g}$ of trypsin gold (Promega, Madison, Wisconsin, USA) per $100 \mu \mathrm{g}$ protein overnight at $37^{\circ} \mathrm{C}$. Filter cartridges were inverted and the peptides were spun off the filter with three washes of $50 \mathrm{mmol} / 1$ Tris or HEPES. Peptides were lyophilized, resuspended in water, and quantified by ultraviolet absorbance at $280 \mathrm{~nm}$. Samples were then frozen at $-80^{\circ} \mathrm{C}$ for future analysis or processing. When isobaric tags for relative and absolute quantitation (iTRAQ) labeling was required, digested peptides were resuspended in $30 \mu \mathrm{l}$ of $100 \mathrm{mmol} / 1$ HEPES. The contents of a single iTRAQ reagent tube (AB Sciex, Framingham, Massachusetts, USA) were resuspended in $70 \mu$ l of ethanol and added to the peptides. The $\mathrm{pH}$ of each reaction was verified and adjusted to $\mathrm{pH} 8$ if necessary for optimal labeling efficiency. After labeling, $100 \mu \mathrm{l}$ of $\mathrm{H}_{2} \mathrm{O}$ was added to quench any further labeling.

In order to ensure equal, one-to-one ratios between all iTRAQ reporter ions and verification of efficient labeling, equal volumes $(1 \mu \mathrm{l})$ of each labeled sample were mixed with buffer A ( $2 \%$ acetonitrile, $0.1 \%$ formic acid) to a final volume of $20 \mu \mathrm{l}$, and analyzed by online $\mathrm{nLC} / \mathrm{MS} / \mathrm{MS}$ (see below). Ratios from the most abundant proteins were then used to make minor corrections to the final mixing volumes ensuring equal $1: 1$ ratios for all reporter channels.

Samples were multiplexed to ensure an overall $1: 1$ ratio for all the iTRAQ reporter channels. $75 \mu \mathrm{g}$ of peptide was lyophilized and resuspended in $200 \mathrm{mmol} / 1$ ammonium formate, $\mathrm{pH} 10$ and loaded onto an offline high-pH reversed phase column for fractionation (Waters, Milford, Massachusetts, USA; XBridge C18 $3.5 \mu \mathrm{m}$ $2.1 \times 100 \mathrm{~mm}$ ) described previously [13]. Samples were fractionated using an Agilent 1200 series microflow pump (Agilent, Santa Clara, California, USA) with buffer A $(20 \mathrm{mmol} / 1$ ammonium formate, $\mathrm{pH} 10)$ and buffer $\mathrm{B}$ 
(90\% ACN, $20 \mathrm{mmol} / 1$ ammonium formate, $\mathrm{pH} 10)$. The samples from each patient were then generally subjected to one of two linear gradients. A non-iTRAQ labeled gradient $(0-60 \%$ buffer $\mathrm{B}$ at $150 \mu \mathrm{l} / \mathrm{min}$ for $66 \mathrm{~min})$ or a iTRAQ specific gradient $(3 \% \mathrm{~B}-10 \mathrm{~min}, 8-11.5 \% \mathrm{~B}-$ $7 \mathrm{~min}, 11.5-60 \% \mathrm{~B}-58 \mathrm{~min}$, at $150 \mu \mathrm{l} / \mathrm{min})$. A total of 24 fractions were collected over the peptide elution profile and then concatenated into 12 final fractions. Fractions were then lyophilized and stored at $-80^{\circ} \mathrm{C}$ for future MS analysis.

Peptide fractions were resuspended in nano-LC buffer A ( $0.1 \%$ formic acid, $2 \% \mathrm{ACN})$ and approximately $1.5 \mu \mathrm{g}$ from each fraction was injected onto a $2-\mathrm{cm}$ precolumn $(\mathrm{id}=100-\mu \mathrm{m}) \quad$ consisting of $5-\mu \mathrm{m}$ particle-sized ReproSil-Pur C18- AQ resin (Dr Maisch GmbH, Beim Bruckle, Germany). Peptides were further resolved on a $10-\mathrm{cm}$ analytical column (id $=75-\mu \mathrm{m})$ consisting of $3-\mu \mathrm{m}$ particle-sized ReproSil-Pur C18-AQ resin (Dr Maisch $\mathrm{GmbH}$ ) using an Easy-nLC system (Thermo Fisher Scientific, Waltham, Massachusetts, USA) with a linear gradient from 0 to $40 \%$ buffer B $(0.1 \%$ formic acid, $98 \% \mathrm{ACN}$ ) over $120 \mathrm{~min}$ at a flow rate of $300 \mathrm{nl} / \mathrm{min}$. The total runtime was $160 \mathrm{~min}$, which included sample loading, gradient elution, ACN wash, and column equilibration. Mass spectra were acquired using a datadependent method using an Orbitrap Velos mass spectrometer (Thermo Fisher Scientific). The top 10 abundant precursor ions from each survey scan were isolated $(2.0 \mathrm{~m} / \mathrm{z}$ isolation width) and fragmented by higher-energy collision dissociation (45\% normalized collision energy, with $100 \mathrm{~ms}$ activation time). The survey scans, were acquired in the Orbitrap using a mass window of $300-1700 \mathrm{~m} / \mathrm{z}$ at a target resolution of 60000 , at $400 \mathrm{~m} / \mathrm{z}$, and the subsequent fragment ion scans were acquired over a dynamic $\mathrm{m} / \mathrm{z}$ range. The lower threshold for selecting a precursor ion for fragmentation was 1000 ions. Dynamic exclusion was enabled using a list size of 500 features, an $\mathrm{m} / \mathrm{z}$ tolerance of $15 \mathrm{ppm}$, a repeat count of 1 , a repeat duration of $30 \mathrm{~s}$, and an exclusion duration of $15 \mathrm{~s}$, with early expiration disabled.

RAW (Thermo Fisher) data files from the Orbitrap Velos were processed using the Mascot search engine (v2.3 Matrix Science, London, UK). All data were searched against the International Protein Index (IPI version 3.78; Ingenuity IPA, Redwood City, California, USA) human database. The following search parameters were used: monoisotopic precursor tolerance was set to $10 \mathrm{ppm}$ while the MS/MS tolerance was set to 0.5 Da. Enzyme specificity was set to trypsin with a maximum of one missed cleavage. Carbamidomethyl of cysteine, iTRAQ labeling of N-termini and lysine's were all applied as fixed modifications. Oxidation of methionine, and iTRAQ labeling of tyrosine were set as variable modifications. All donors were imported into an instance of Trans Proteomic Pipeline implemented in Scaffold v3.4.9 (Proteome Software, Portland, Oregon, USA).
X!Tandem (thegpm.org) was used within Scaffold as an additional measure for confident peptide and protein identifications (thegpm.org; version CYCLONE, 2010.12.01.1). Quantitative results were exported from Scaffold with the following criteria: minimum peptide probability of $80 \%$ (Peptide Prophet; Protein \& Peptide Prophet, Seattle, Washington, USA) a minimum protein probability of $99 \%$ (Protein Prophet), and a minimum of two peptides per protein. These setting resulted in a $0.1 \%$ protein and $1.5 \%$ peptide false discovery rate for the entire dataset. Statistical significance between the respective study groups was evaluated using a Student's $t$-test for each protein comparison.

\section{Western blot analysis}

PBMC lysate $(25 \mu \mathrm{g})$ was loaded on to a NuPAGE 4-12\% Bis-Tris precast acrylamide gel (Life Technologies). Affinity MagicMarkTM XP (Life Technologies) was also loaded as molecular weight marker. Gels were resolved to completion using an XCell SureLock MiniCell (Invitrogen, Carlsbad, California, USA) electrophoresis tank running at $150 \mathrm{~V}$. After protein gel electrophoresis, proteins were transferred onto a nitrocellulose membrane using the Invitrogen iBlot (Invitrogen) transfer system. Membranes were directly placed in Odyssey blocking buffer (OBB; LI-CORE) for $1 \mathrm{~h}$ at room temperature with gentle mixing. Primary antibodies Mx1, RGS6, and HLA-C (Epitomics) were diluted in $\mathrm{OBB}$ with the addition of $0.1 \%$ Tween-20 (Sigma) and incubated overnight at $4{ }^{\circ} \mathrm{C}$ on an orbital shaker. An antibody loading control ( $\beta$-Actin; Abcam) incubated for $1 \mathrm{~h}$ at room temperature followed by four 5-min washes with phosphate-buffered saline-Tween (PBS, 0.1\% Tween-20). Secondary fluorescent antibodies (IRDye 680R/800CW, diluted in OBB, 0.1\% Tween 20, and $0.01 \%$ SDS; LI-CORE) were then incubated at room temperature for $1 \mathrm{~h}$ in the dark followed by another four 5-min washes with PBST. A final wash of PBS for $5 \mathrm{~min}$ was performed. Blots were subsequently dried at $4^{\circ} \mathrm{C}$ and imaged using an Odyssey CLx infrared imagining system (LI-CORE).

\section{Mx1 and Mx2 ELISA}

A commercial ELISA was purchased from USCN Life Science Inc. (Wuhan, China) for the quantification of $\mathrm{Mx} 1$ and $\mathrm{Mx} 2$ in PBMC lysate. Briefly, a standard doubling dilution series was prepared for either $\mathrm{Mx} 1$ or Mx2 (20-0.02 ng/ml) and PBMC lysate samples in PBS from each study participant were added to the plates in duplicate. The plates were sealed and incubated for $2 \mathrm{~h}$ at $37^{\circ} \mathrm{C}$. After incubation the samples and standard curve were aspirated from the plate using the Bioteck plate washer. A biotin-conjugated antibody specific for Mx1 or Mx2 was added (1:100 dilution) followed by incubation at $37^{\circ} \mathrm{C}$ for $60 \mathrm{~min}$. After incubation the plates were manually washed three times with the wash buffer provided in the ELISA kit. A secondary avidin conjugated 
to horseradish peroxidase was then added $(1: 100$ dilution) and incubated for $30 \mathrm{~min}$ at $37^{\circ} \mathrm{C}$. The plates were again washed five times with the provided wash buffer. Tetramethylbenzidine substrate was then added to the plates and incubated at $37^{\circ} \mathrm{C}$ for $15-30 \mathrm{~min}$. Stop solution (sulphuric acid) was then added to terminate the reaction. The plates were read using the SpectraMax Plus spectrophotometer at a wavelength of $450 \mathrm{~nm}$. A fourparameter curve was used to fit the standard curve for accurate quantitation of $\mathrm{Mx} 1$ and $\mathrm{Mx} 2$.

\section{Epidemiological analysis}

The Mx1 and Mx2 expression levels measured by the commercial ELISA were transformed using their natural logarithms. The log-transformed values of $\mathrm{Mx} 1$ and $\mathrm{Mx} 2$ were subsequently used as outcome variables in separate multiple linear regression models. Where appropriate, $\mathrm{t}-$ tests and ANOVA's were used in bivariate analyses with logged values of $\mathrm{Mx} 1 / \mathrm{Mx} 2$ used as the outcome variables. Multiple linear regression models were fitted to the data and used to assess the independent association of HESN status and the logged values of Mx1 and Mx2, adjusted for age, recent infections (2 months) and DepoProvera. Given their theoretical importance, all confounders were kept in full linear regression models; however, each confounder was entered separately in the following order: use of Depo-Provera, age, and recent infection in the last 2 months. In addition, the interaction between Depo-Provera use and HESN status was added as an additional variable in the final full model. All tests performed were considered statistically significant at $P<0.05$. Stata 12 (College Station, Texas, USA) was used for all epidemiological analyses. Age, recent infections, and Depo-Provera were included a priori due to the impact of these variables on general immune function. The interaction between HESN status and Depo-Provera was also planned a priori due to our interest in whether or not levels of $\mathrm{Mx} 1 / \mathrm{Mx} 2$ between HESN and high-risk negative controls differed by use of Depo-Provera. From an initial sample of 202 women, 190 had detectable levels for both Mx1 and Mx2. For all models, menopausal $(n=2)$, pregnant $(n=5)$ and missing values for contraception use $(n=22)$ were excluded from the analyses, leaving a total sample size of 161 women.

\section{Discussion}

Although still controversial [14], several studies have linked DMPA use with increased risk of HIV acquisition $[15,16]$. These studies suggest that HESN women using DMPA are likely at a higher risk of infection than HESN women who do not use DMPA. The high levels of Mx2 observed in HESN women, epidemiologically resistant to HIV, may provide a plausible biologic mechanism to explain how this particular subset of HESN women is protected from infection. DMPA is commonly used in sub-Saharan Africa and represents a relatively inexpensive alternative to oral or intrauterine device contraception. For this reason, it is possible that DMPA use could be confounded by some other epidemiological factor not captured in our study. However, given that DMPA has been shown to suppress interferon responses, and that interferon-stimulated genes, such as $\mathrm{Mx} 2$, have been associated with protection from SIV infection in rhesus macaques, a biological interaction between DMPA and Mx2 in HESN women is plausible [17,18]. Although it is tempting to suggest that DMPA alone could be used to modulate resistance, its broad immunosuppressive effects and reported increase in HIV acquisition risk will most likely eliminate its use in preventive strategies. However, identification of an intermediate biologic link between DMPA and innate Mx2 signaling in HESN women may allow for targeted regulation of $\mathrm{Mx} 2$ as a novel prevention strategy. Interventions such as RNA interference of known regulators of $\mathrm{Mx} 2$ or through as yet defined viral/ host mRNA mechanisms could alternatively be used to regulate HIV resistance and susceptibility via Mx2. Data from our group and others have shown that the HESN phenotype is multifactorial; however, one commonality is the altered expression of innate factors limiting HIV replication, immune activation, and the recruitment of target cells [3]. Increased expression of $\mathrm{Mx} 2$ in HESN women using DMPA may suggest a link to this immune quiescent phenotype; however, a causal relationship between altered interferon signaling and the genetic regulation of $\mathrm{Mx} 2$, influenced by hormonal contraception, needs to be established. One recent study has suggested that $\mathrm{Mx} 2$ expression of injection drug users and sexually exposed HIV individuals may play a role in altered susceptibility to HIV infection [19]. Thus, further studies of the relationship between HIV susceptibility, Mx2 expression, and DMPA use are clearly warranted. These data represent the first report of $\mathrm{Mx} 2$ as a contributor to altered susceptibility to HIV infection in HESN female sex workers in the context of epidemiologic confounders such as hormonal contraception use. Future studies need to identify mechanisms that may regulate higher expression of $\mathrm{Mx} 2$ in HESN women and its interaction with DMPA. A deeper understanding of interferon responses to HIV in the real-world setting could lead to HIV preventative measures that make use of Mx2 to modulate resistance and susceptibility to HIV infection.

\section{Acknowledgements}

This research was supported by the Canadian Institute for Health Research (C.I.H.R.), the National Institutes of Health through the HIV Vaccine Trials Network (H.V.T.N.) and the Public Health Agency of Canada (P.H.A.C.). D.S. was supported by a C.I.H.R. Canada Graduate Scholarship and by a C.I.H.R. International Infectious Disease and Global Health Training Program Fellowship. The author would also like to acknowledge 
the support of the P.H.A.C. Mass Spectrometry \& Proteomics Core Facility, without which our studies would not be possible.

Author contributions: D.R.S., K.R.F., F.A.P., and T.B.B. designed the study and wrote the manuscript. D.R.S carried out the experiments. Sample preparation and technical expertise was provided by M.A., G.R.W. and S.J.M. provided mass spectrometry expertise and equipment. S.Y.S. and L.R.M. facilitated the epidemiological analysis. All authors read and approved the final manuscript.

\section{Conflicts of interest}

There are no conflicts of interest.

\section{References}

1. Fowke KR, Nagelkerke NJ, Kimani J, Simonsen JN, Anzala AO, Bwayo JJ, et al. Resistance to HIV-1 infection among persistently seronegative prostitutes in Nairobi, Kenya. Lancet 1996; 348:1347-1351.

2. Ball TB, Ji H, Kimani J, Mclaren P, Marlin C, Hill AVS, et al. Polymorphisms in IRF-1 associated with resistance to HIV-1 infection in highly exposed uninfected Kenyan sex workers. AIDS 2007; 21:1091-1101.

3. Card CM, Ball TB, Fowke KR. Immune Quiescence: a model of protection against HIV infection. Retrovirology 2013; 10:141.

4. Burgener A, Rahman S, Ahmad R, Lajoie J, Ramdahin S, Mesa C, et al. Comprehensive proteomic study identifies serpin and cystatin antiproteases as novel correlates of HIV-1 resistance in the cervicovaginal mucosa of female sex workers. / Proteome Res 2011; 10:5139-5149.

5. Stein DR, Burgener A, Ball TB. Proteomics as a novel HIV immune monitoring tool. Curr Opin HIV AIDS 2013; 8:140-146.

6. Su RC, Sivro A, Kimani J, Jaoko W, Plummer FA, Ball TB. Epigenetic control of IRF1 responses in HIV-exposed seronegative versus HIV-susceptible individuals. Blood 2011; 117:26492657.
7. Sandler NG, Bosinger SE, Estes JD, Zhu RTR, Tharp GK, Boritz E, et al. Type $\mathbf{I}$ interferon responses in rhesus macaques prevent SIV infection and slow disease progression. Nature 2014; 511:601-605.

8. Kane M, Yadav SS, Bitzegeio J, Kutluay SB, Zang T, Wilson SJ, et al. MX2 is an interferon-induced inhibitor of HIV-1 infection. Nature 2013; 502:563-566.

9. Goujon C, Moncorgé O, Bauby H, Doyle T, Ward CC, Schaller $\mathrm{T}$, et al. Human MX2 is an interferon-induced postentry inhibitor of HIV-1 infection. Nature 2013; 502:559-562.

10. Liu Z, Pan Q, Ding S, Qian J, Xu F, Zhou J, et al. The InterferonInducible MxB Protein Inhibits HIV-1 Infection. Cell Host Microbe 2013; 14:398-410.

11. Haller O. Dynamins are forever: MxB inhibits HIV-1. Cell Host Microbe 2013; 14:371-373.

12. Wiśniewski JR, Zougman A, Nagaraj N, Mann M. Universal sample preparation method for proteome analysis. Nat Meth 2009; 6:359-362.

13. Stein DR, Hu X, McCorrister SJ, Westmacott GR, Plummer FA, Ball $\mathrm{TB}$, et al. High pH reversed-phase chromatography as a superior fractionation scheme compared to off-gel isoelectric focusing for complex proteome analysis. Proteomics 2013; 13:2956-2966.

14. McCoy SI, Zheng W, Montgomery ET, Blanchard K, van der Straten A, De Bruyn G, et al. Oral and injectable contraception use and risk of HIV acquisition among women in sub-Saharan Africa. AIDS 2013; 27:1001-1009.

15. Morrison CS, Chen P-L, Kwok C, Richardson BA, Chipato T, Mugerwa R, et al. Hormonal contraception and HIV acquisition: reanalysis using marginal structural modeling. AIDS 2010; 24:1778-1781.

16. Heffron R, Donnell D, Rees H, Celum C, Mugo N, Were E, et al. Use of hormonal contraceptives and risk of HIV-1 transmission: a prospective cohort study. Lancet Infect Dis 2012; 12:1926.

17. Hughes GC, Thomas S, Li C, Kaja M-K, Clark EA. Cutting edge: progesterone regulates IFN-alpha production by plasmacytoid dendritic cells. J Immunol 2008; 180:2029-2033.

18. Tayel SS, Helmy AA, Ahmed R, Esmat G, Hamdi N, Abdelaziz Al. Progesterone suppresses interferon signaling by repressing TLR-7 and MxA expression in peripheral blood mononuclear cells of patients infected with hepatitis C virus. Arch Virol 2013; 158:1755-1764.

19. Sironi M, Biasin M, Cagliani R, Gnudi F, Saulle I, Ibba S, et al. Evolutionary analysis identifies an MX2 haplotype associated with natural resistance to HIV-1 infection. Mol Biol Evol 2014; 31:2402-2414. 